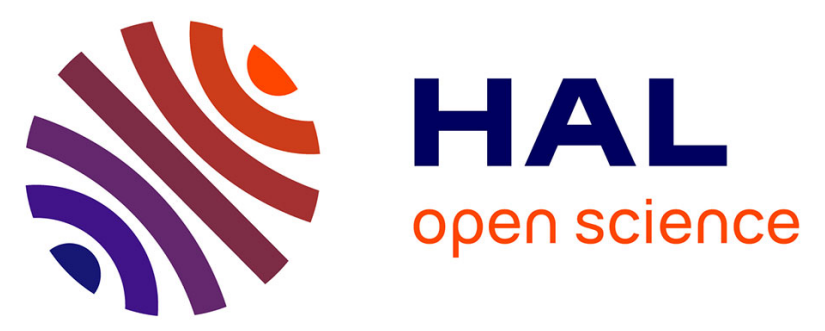

\title{
Le partage de la valeur économique entre les acteurs d'un projet de télémédecine : enjeux méthodologiques ?
}

Camille Jean, Tu-Anh Duong, Julie Stal-Le Cardinal, Marija Jankovic, Jean-Claude Bocquet, Pierre Espinoza

\section{- To cite this version:}

Camille Jean, Tu-Anh Duong, Julie Stal-Le Cardinal, Marija Jankovic, Jean-Claude Bocquet, et al.. Le partage de la valeur économique entre les acteurs d'un projet de télémédecine : enjeux méthodologiques ?. European Research in Telemedicine / La Recherche Européenne en Télémédecine, 2016, 5 (2), pp.37-44. 10.1016/j.eurtel.2016.04.002 . hal-01358709

\section{HAL Id: hal-01358709 \\ https://hal.science/hal-01358709}

Submitted on 1 Sep 2016

HAL is a multi-disciplinary open access archive for the deposit and dissemination of scientific research documents, whether they are published or not. The documents may come from teaching and research institutions in France or abroad, or from public or private research centers.
L'archive ouverte pluridisciplinaire HAL, est destinée au dépôt et à la diffusion de documents scientifiques de niveau recherche, publiés ou non, émanant des établissements d'enseignement et de recherche français ou étrangers, des laboratoires publics ou privés. 


\title{
Le partage de la valeur économique entre les acteurs d'un projet de télémédecine : enjeux méthodologiques?
}

\author{
Camille Jean ${ }^{1}$, Tu-Anh Duong ${ }^{2,3}$, Julie Stal-Le Cardinal ${ }^{2}$, Marija Jankovic ${ }^{2}$, \\ Jean-Claude Bocquet ${ }^{2}$, Pierre Espinoza ${ }^{4}$
}

\author{
${ }^{1}$ École Arts et Métiers, Laboratoire Conception de Produits et Innovation, Paris, France \\ 2École Centrale Paris, Laboratoire Génie Industriel, Châtenay-Malabry, France \\ ${ }^{3}$ Hôpital Henri Mondor, AP-HP, Département de dermatologie, Créteil, France \\ ${ }^{4}$ Mutualité française des Côtes d'Armor, Saint-Brieuc, France
}

Corresponding author: Camille Jean

\begin{abstract}
Résumé :
Introduction : La pérennité des projets de télémédecine est trop souvent remise en cause par l'absence de développement préalable de modèles économiques durables qui permettraient d'élaborer de nouvelles organisations à même d'équilibrer le financement de leur déploiement et de leur fonctionnement. Les méthodes d'évaluation médico-économiques et les méthodes multicritères existantes ne permettant pas de répondre à cette problématique, une nouvelle méthode est donc nécessaire.
\end{abstract}

Matériels et Méthodes : Les outils d'analyse des systèmes complexes utilisés dans le domaine du Génie Industriel sont mobilisés pour créer cette méthode. La perspective holiste adoptée est l'occasion d'élaborer des modèles économiques plus durables pour chacun des acteurs d'un projet de télémédecine.

Résultats : La méthode proposée permet de créer des scenarii variés de modèles économiques et offre une vision précise de ce que l'évolution projetée aura comme impact sur chacun des acteurs impliqués et sur le système. Un exemple d'application est détaillé.

Discussion et Conclusions : La méthode présentée permet de concevoir des scenarii de partage de la valeur économique entre les acteurs d'un projet de télémédecine. Son application à un projet particulier permet de servir de support de discussion lors des négociations entre acteurs concernés. Elle permet également d'évaluer la pérennité du projet de télémédecine ainsi négocié.

Mots clés : Modèle économique, partage de la valeur, système complexe, innovation, conception 


\title{
Sharing economic value between the stakeholders of a telehealth project: methodological issues?
}

\author{
Camille Jean ${ }^{1}$, Tu-Anh Duong ${ }^{2,3}$, Julie Stal-Le Cardinal ${ }^{2}$, Marija Jankovic ${ }^{2}$, \\ Jean-Claude Bocquet ${ }^{2}$, Pierre Espinoza ${ }^{4}$
}

\author{
1École Arts et Métiers, Laboratoire Conception de Produits et Innovation, Paris, France \\ 2École Centrale Paris, Laboratoire Génie Industriel, Châtenay-Malabry, France \\ ${ }^{3}$ Hôpital Henri Mondor, AP-HP, Département de dermatologie, Créteil, France \\ ${ }^{4}$ Mutualité française des Côtes d'Armor, Saint-Brieuc, France
}

Corresponding author: Camille Jean

\begin{abstract}
:
Introduction: The durability of telehealth projects is too often questioned by the lack of prior development of sustainable business models allowing the finding of the equilibrium of the funding for the deployment and for the operational phases. The existing medico-economic and multidisciplinary methods do not address this problem; hence a new one is needed.

Materials and Methods: The complex system design tools used in the field of Industrial Engineering are used as a base for this new method. The holistic approach adopted represents an opportunity to develop sustainable business models taking into account needs and viewpoints of each stakeholder of a telehealth project.

Results: The proposed method aims at creating various scenarios of business models and offering a clear vision on how the proposed changes will impact each of the stakeholders involved as well as the overall system. An example of how this can be applied is detailed.

Discussion and Conclusions: The presented method allows the design of economic value sharing scenarios between all the stakeholders of a telehealth project. Its application to a particular one can act as a basis for discussions during negotiations between stakeholders. It can also support the assessment of the overall project's sustainability.
\end{abstract}

Keywords: Business model, economic value sharing, complex system, innovation, design engineering 


\section{Introduction}

Un des enjeux d'un projet de télémédecine est de parvenir à un modèle organisationnel valide, créateur de valeurs et économiquement pérenne [1-3]. Les études existantes incluant un aspect économique se focalisent généralement sur l'analyse de l'efficience de projets particuliers de télémédecine [4]. Elles comparent les coûts et les résultats (les créations de valeurs) avant puis après l'installation d'un projet dans l'objectif de développer une aide à la décision publique de son extension à grande échelle.

Cependant, démontrer la pertinence d'un projet expérimental à l'échelle locale ne permet pas nécessairement d'en déduire sa validité à une échelle d'intervention plus large; le nombre d'acteurs et de paramètres à prendre en compte varie de fait considérablement en fonction de l'échelle d'intervention retenue. Afin de surmonter cette difficulté, il est souhaitable de proposer à l'ensemble des parties prenantes impliquées dans l'extension du projet retenu des modèles de partage de la valeur viables et pérennes.

Pour ce faire, la première partie de cet article décrit les méthodes d'évaluation médico-économiques existantes concernant la télémédecine et précise leurs perspectives, tandis que la seconde détaille les outils d'analyse des systèmes complexes utilisés dans le domaine du Génie Industriel qui sont mobilisés pour concevoir la méthode préconisée. La troisième partie la détaille et la quatrième l'illustre avec un exemple.

\section{Matériels et Méthodes}

\subsection{Analyse des méthodes d'évaluations médico-économiques existantes}

Les méthodes d'évaluation de l'efficience en santé avaient initialement pour vocation de comparer différentes thérapies pour sélectionner celle qui apporterait le plus de bénéfices par rapport aux coûts. Par la suite, ces méthodes ont été utilisées pour aider à choisir parmi différentes stratégies d'organisation de santé. La figure 1 illustre les quatre méthodes les plus utilisées en santé [5].

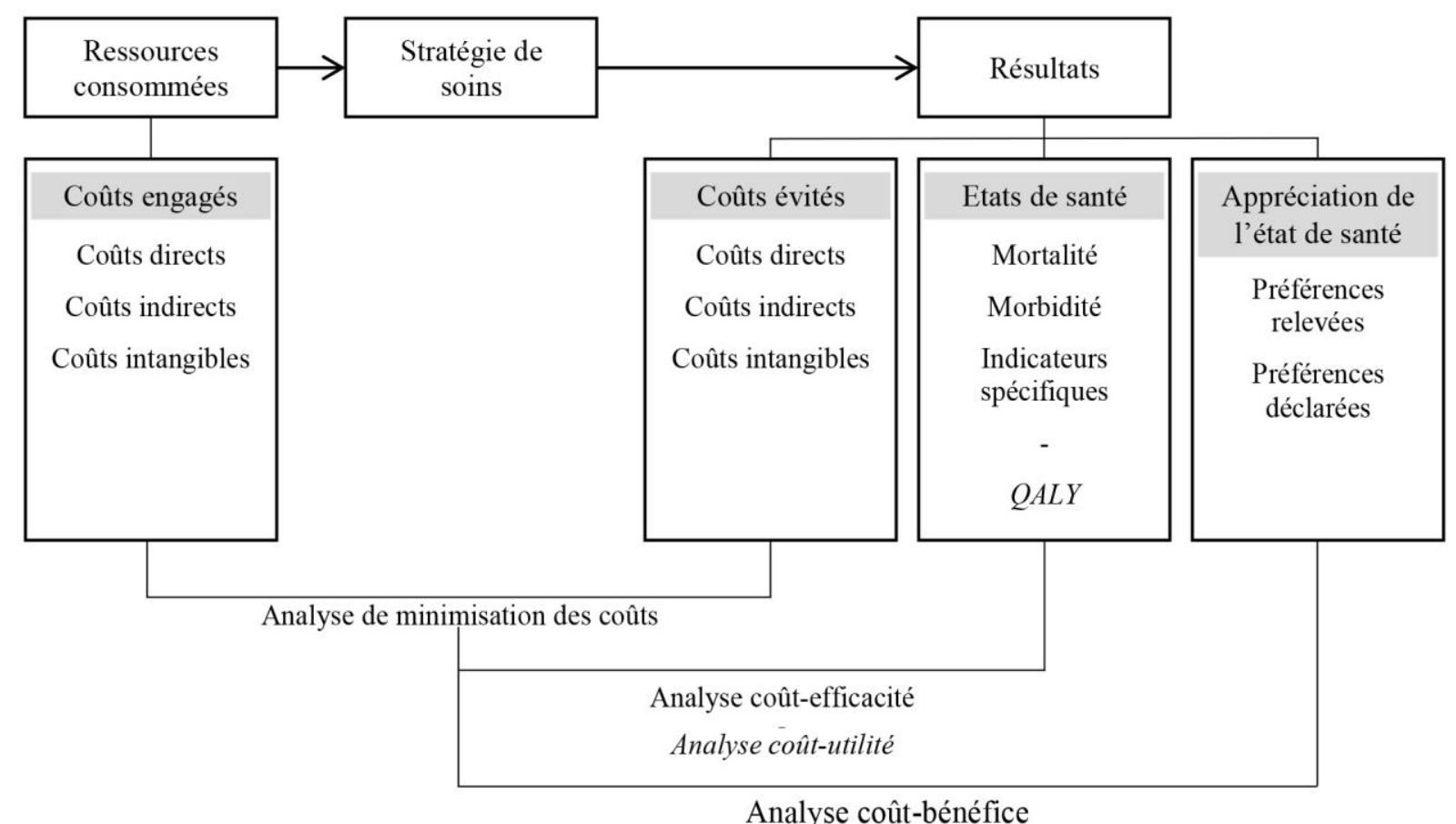

Figure 1 : Méthodes disponibles pour évaluer l'efficience d'une stratégie de soin (schéma adapté de [6]) Figure 1: Methods available to evaluate the effectiveness of a care strategy (figure adapted from [6]) 
Ces quatre méthodes sont :

- l'analyse de minimisation des coûts (AMC) comparant le coût de différents projets quand ils ont exactement les mêmes conséquences ;

- l'analyse coût-efficacité (ACE) comparant le coût et les résultats de différents projets grâce à des indicateurs dépassant la simple mesure de leurs conséquences financières ;

- l'analyse coût-utilité (ACU) comparant le coût et les résultats de différents projets grâce à l'indicateur du gain d'années de vie ajustées par la qualité (QALY) ;

- l'analyse coût-bénéfice (ACB) comparant le coût et les résultats de différents projets sans affecter une valeur monétaire aux résultats et permettant d'apprécier la valeur sociale d'une dépense publique.

Sur ces quatre méthodes, seules les trois premières (AMC, ACE et $A C U$ ) sont préconisées par la Haute Autorité de Santé (HAS) française pour évaluer des projets de santé et ainsi éclairer la décision publique d'allocation de ressources [7].

Dans son rapport de 2013, la HAS fournit un riche état de l'art des études existantes sur l'efficience de la télémédecine en France et dans le monde entre les années 2000 et 2012 [4]. Après l'analyse de 286 références, elle conclut en indiquant que les activités de télémédecine sont à forte composante organisationnelle, largement dépendante du contexte et de la problématique médicale. Ainsi, les résultats sont faiblement généralisables et l'efficience dépend de chaque projet.

Comme le souligne Pascal [8], les méthodes classiques d'évaluation médico-économique ne sont pas adaptées pour évaluer un projet de télémédecine en phase de démarrage. II existe des méthodes plus appropriées, notamment des évaluations multicritères qui permettent de juger plus globalement l'impact d'un dispositif [9]. Le projet MAST [10] s'inscrit dans ces méthodes et propose une grille d'évaluation multidisciplinaire en sept domaines: problèmes de santé, sécurité, efficacité, perspective des patients, aspects économiques, aspects organisationnels, aspects socioculturels, éthiques et légaux. Sur les aspects économiques, cette grille propose une évaluation uniquement circonscrit à deux points de vue : (1) celui du système global de santé en comparant un projet de télémédecine avec une alternative pertinente, en réalisant par exemple une ACE, et (2) celui de l'entité qui porte le projet en réalisant une étude de retour sur investissement (ROI).

Ainsi, que cela soit avec des méthodes classiques d'évaluation médico-économique ou avec des méthodes multicritères, la pertinence économique des projets n'est évaluée que sur la base de quelques parties prenantes ou du système global de santé. Ce constat est confirmé par l'étude de Bergmo [11]. Sa revue de la littérature réalisée sur 33 articles scientifiques internationaux montre que les deux tiers d'entre eux ne se préoccupent que de la valeur ajoutée de la télémédecine pour le système de santé dans son ensemble, tandis que le dernier tiers n'offre au maximum que deux perspectives. La revue systématique de la littérature d'Ekeland et al. [12] basée sur l'analyse de 80 articles confirme également cette restriction.

Les méthodes évoquées précédemment ne permettent donc pas d'appréhender les aspects économiques du point de vue de chacune des parties prenantes impliquées dans le projet. De plus, elles n'aident ni à concevoir de modèles économiques alternatifs ni à évaluer leur pertinence pour chacune des parties prenantes.

Le paragraphe suivant explore les outils d'analyse des systèmes complexes utilisés dans le domaine du Génie Industriel permettant de répondre à cette problématique.

\subsection{Outils d'analyse des systèmes complexes utilisés dans le domaine du Génie Industriel}

Les projets de télémédecine peuvent être considérés comme des systèmes complexes. En effet, ils sont constitués d'entités (individus, organisations) dont la taille, la sensibilité au contexte, la variété des éléments et l'interdépendance que ces derniers entretiennent entre eux, rendent impossibles à circonscrire totalement et donc à être entièrement prévisibles. Dès lors, les approches systémique et constructiviste proposées par Simon [13] et Le Moigne [14] permettent de les modéliser pour mieux les concevoir.

Ainsi, un projet de télémédecine est conçu en définissant, en fonction du temps, ses objectifs, ses processus et ses ressources. Une fois les processus définis et justifiés par leurs créations de valeurs, l'objectif est de les rendre viables. Les différentes possibilités de rémunération des parties prenantes doivent, par conséquent, 
être analysées, modélisées et simulées afin d'éclairer les choix des décideurs quant aux différentes alternatives possibles de modèles économiques [15].

Dans cet optique, Porter [16] a travaillé sur le partage de la valeur ajoutée dans un écosystème d'entreprise. Cette valeur provient du client et est repartie entre les employés, les banques, les collectivités, les fournisseurs et les actionnaires.

En élargissant le périmètre à des écosystèmes multi-entreprises, des travaux ont été réalisés sur le partage de la valeur ajoutée dans une chaine linéaire d'acteurs. Par exemple, dans l'industrie agroalimentaire (répartition des marges par filières) [17], le tourisme (avec les questions sur la rétribution des nouveaux acteurs intermédiaires) [18] et le livre numérique (avec le repositionnement d'acteurs technologiques dans l'industrie du livre) [19].

Cependant, aucune étude concernant le partage de la valeur ajoutée au sein d'un réseau complexe d'acteurs tel que celui d'un projet de télémédecine n'a été remarquée.

Basé sur ce constat et s'inspirant des outils d'analyse présentés précédemment, une nouvelle méthode permettant de concevoir des modèles économiques est proposée dans le paragraphe suivant.

\subsection{Proposition d'une nouvelle méthode permettant de concevoir des modèles économiques}

L'objectif de la méthode est de concevoir un nouveau partage durable de la valeur économique entre les différentes parties prenantes d'un projet de télémédecine. Elle se base sur un travail collaboratif à effectuer avec les acteurs de terrain afin de simuler des hypothèses crédibles de flux financiers et de vérifier si les modèles obtenus sont pérennes.

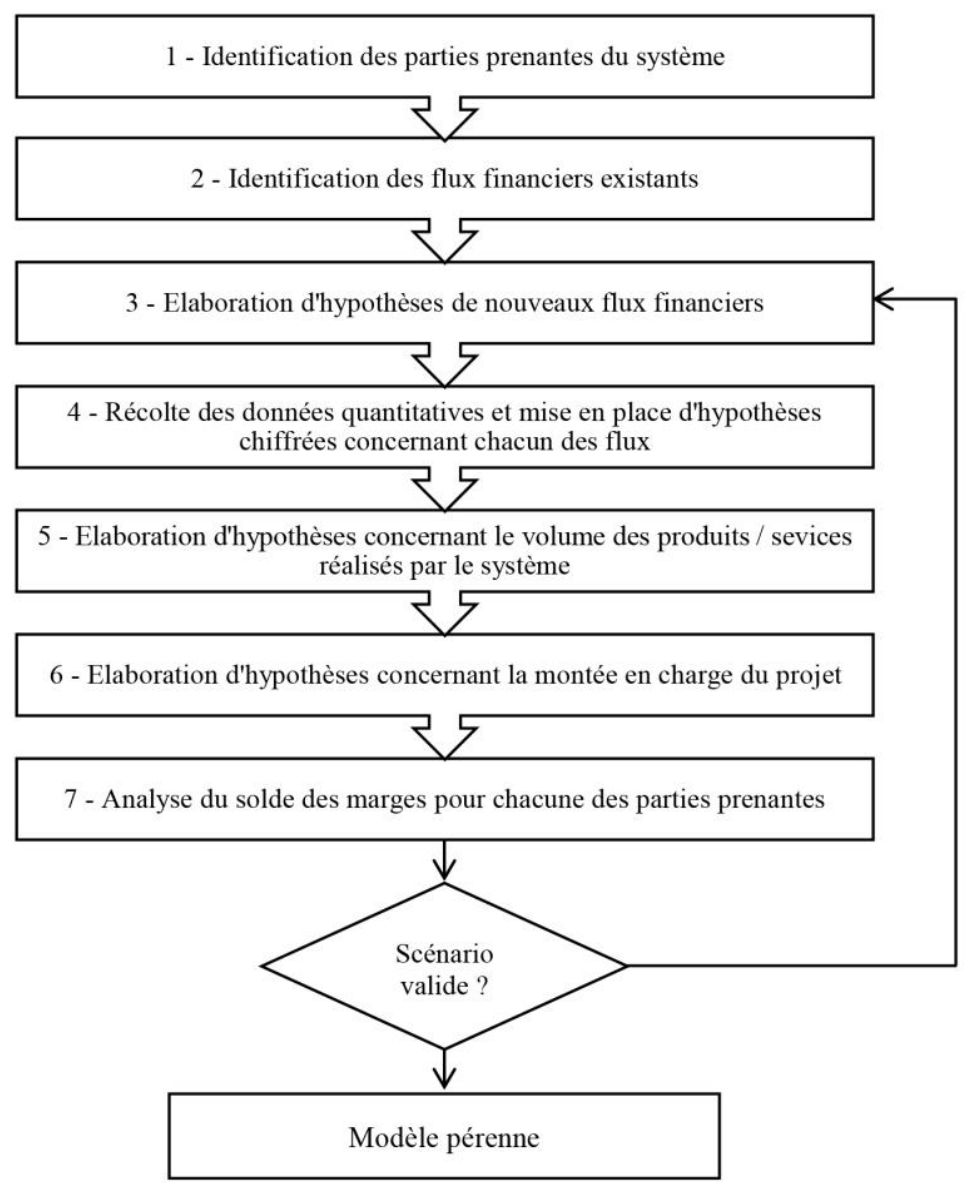

Figure 2 : Méthode de construction de modèle de partage de la valeur économique Figure 2: Method to design models of sharing economic value 
Telles qu'indiquées dans la figure 2, la première et la deuxième étape de la méthode concernent l'identification des parties prenantes du système et des flux financiers existants. La troisième et la quatrième étape permettent l'élaboration des hypothèses de flux financiers et leur quantification via la récolte de données et l'élaboration d'hypothèses quantitatives. La cinquième et la sixième étape permettent d'inclure des hypothèses de volumétrie et de montée en charge. La septième et dernière étape consiste à calculer le solde des marges annuelles pour toutes les parties prenantes. Cet indicateur correspond au bilan de ce que gagne ou perd chacune d'entre elles avec la mise en place du nouveau système. Ainsi, pour une partie prenante $\mathrm{x}$ du système, la marge $\mathrm{M}_{\text {sans }}(x)$ corespond à sa marge annuelle sans la mise en place du nouveau système. La marge $\mathrm{M}_{\mathrm{avec}}(x)$ correspond à celle avec sa mise en place. Le solde des marges correspond à la différence des deux précédentes $\mathrm{M}_{\mathrm{avec}}(x)-\mathrm{M}_{\text {sans }}(x)$.

Ces sept étapes se concluent par l'analyse de la validité du modèle créé, en d'autres termes par l'analyse de sa pérennité. Si le modèle ne l'est pas, il est nécessaire de retourner à l'étape 3 et d'effectuer de nouvelles hypothèses jusqu'à ce que le scénario réalisé soit valide.

L'objectif principal de cette méthode est de promouvoir un dialogue argumenté avec les acteurs de terrain en comparant l'ensemble des scénarii valides pour faire en sorte que le plus satisfaisant soit sélectionné.

\section{Résultats : Exemple d'application de la méthode}

Le projet Télégéria lle-de-France a pour ambition de réaliser 2500 téléconsultations et télé-expertises par an entre des hôpitaux et des établissements d'hébergement pour personnes âgées dépendante (EHPAD). II constitue l'extension d'une expérience locale de télémédecine ayant réalisé plus de 1500 actes de télémédecine [20].

Les travaux de Jean [15] ont permis d'appliquer la méthode proposée dans cet article sur ce projet. Un extrait de ces travaux est présenté, étape par étape, ci-dessous :

\section{Etape 1 : identification des parties prenantes}

Le tableau 1 recense les parties prenantes du projet.

\begin{tabular}{|c|c|}
\hline Partie prenante & Description complémentaire \\
\hline Société & Ensemble des individus de la société française \\
\hline Etat & $\begin{array}{l}\text { Ministères concernés par la télémédecine (Ministère des Affaires sociales et de la Santé, } \\
\text { Ministère de l’Economie et des Finances, ...) }\end{array}$ \\
\hline Collectivités locales & Communes, Départements et Région concernés \\
\hline ARS & Agence régionale de santé \\
\hline Assurance Maladie & Assurance Maladie \\
\hline Autres financeurs & Caisse des Dépôts, Conseils Généraux, Conseils Régionaux, collectivités locales \\
\hline $\begin{array}{l}\text { Mutuelles et } \\
\text { Assurances }\end{array}$ & Mutuelles et Assurances des patients \\
\hline Patients & Ensemble des patients nécessitant une consultation spécialisée \\
\hline $\begin{array}{l}\text { Etablissements } \\
\text { requérants }\end{array}$ & Etablissements de soins requérants tels que les Centres Hospitaliers, les EHPAD ou les SSR \\
\hline Etablissements requis & Etablissements requis proposant un service télémédecine \\
\hline Médecins requérants & Médecins des établissements requérants \\
\hline Médecins requis & Médecins spécialistes requis des établissements pivots \\
\hline Personnels de santé & Personnels de santé des établissements de santé (ex : assistante de télémédecine) \\
\hline $\begin{array}{l}\text { Personnels } \\
\text { administratifs }\end{array}$ & Personnels administratifs des établissements de santé (ex : secrétaire médicale) \\
\hline Ambulanciers & Compagnies d'ambulances gérant une flotte d'ambulances ou de VSL \\
\hline
\end{tabular}




\begin{tabular}{|l|l|}
\hline Entité organisatrice & Gestionnaire du projet \\
\hline $\begin{array}{l}\text { Industriels } \\
\text { (plateforme) }\end{array}$ & Industriels chargés de la recherche et développement de la plateforme technologique \\
\hline Industriels (matériel) & Fournisseurs de matériels techniques et de maintenance \\
\hline
\end{tabular}

Tableau 1 : Parties prenantes retenues pour l'analyse

Table 1: Stakeholders used in the analysis

Après avoir identifié l'ensemble des parties prenantes, les flux financiers existant entre chacune d'elles sont modélisés.

\section{Etapes 2 et 3 : détail des relations financières entre les parties prenantes}

Les parties prenantes du système ont des relations financières les unes avec les autres. La figure 3 les représente sous la forme d'un graphique.

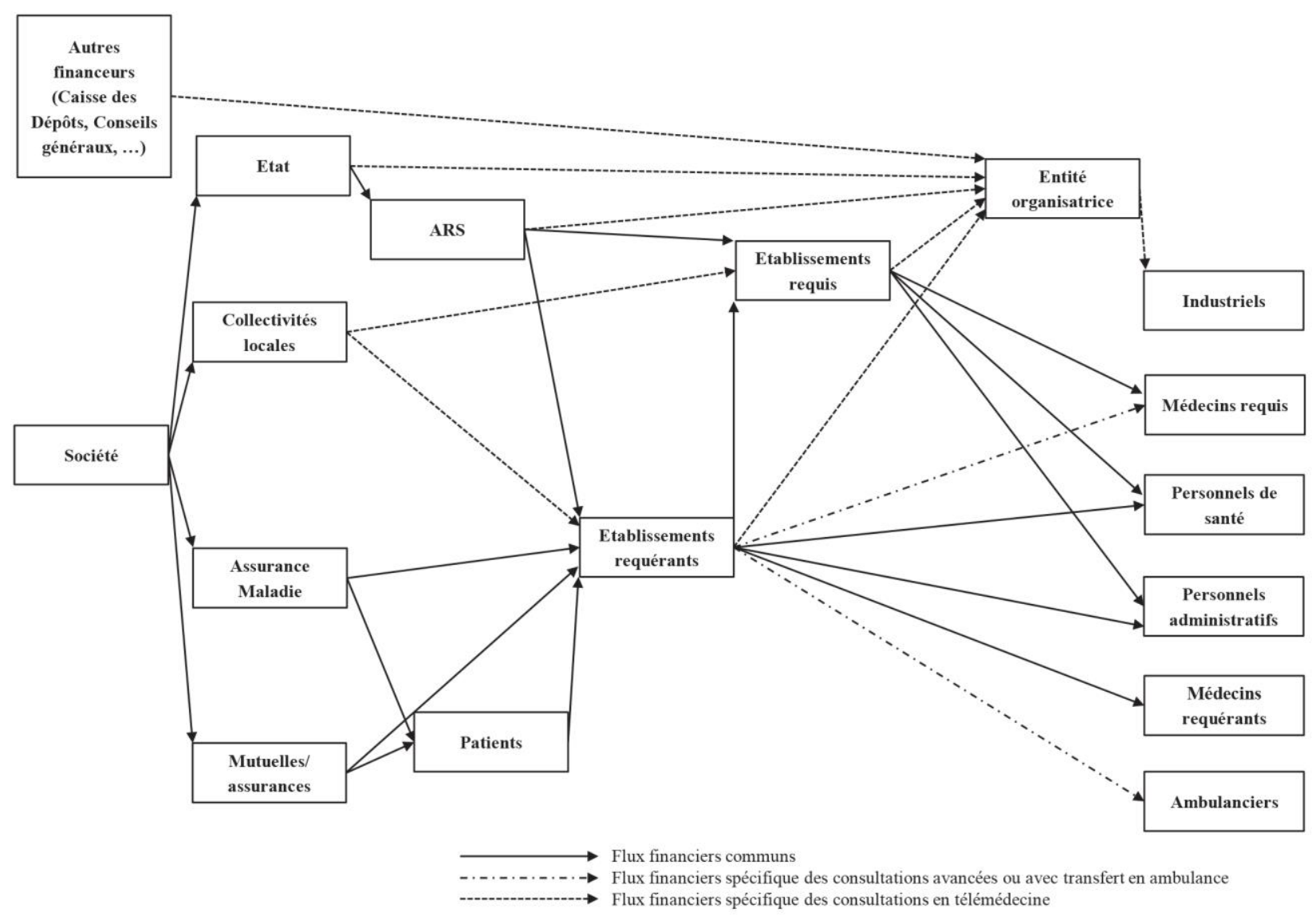

Figure 3 : Exemple de flux financiers entre les parties prenantes

Figure 3: Example of financial flows between stakeholders

Trois types de flux financiers sont indiqués : (1) les flux qui n'ont pas vocation à être modifiés, (2) les flux existants sans la mise en place du projet de télémédecine (transferts de patient en ambulance et consultations avancées) et (3) les flux mis en place lors du déploiement du projet de télémédecine. L'intensité de ces flux varie au cours du temps en fonction de l'étape de déploiement du projet. Ainsi, tous les flux n'ont pas vocation à perdurer dans le temps.

II existe de nombreux autres modèles possibles. Sur celui présenté sur la figure 3, l'hypothèse d'une entité organisatrice (tel un GCS) fédérant l'ensemble des activités de télémédecine d'un territoire a été faite. Cet acteur permettrait d'assurer une coordination administrative et technique des projets de télémédecine. 


\section{Etape 4 à 6 : mise en place des données et des hypothèses chiffrées}

Pour chacune des parties prenantes, une quantification des flux financiers via la récolte de données et l'élaboration d'hypothèses chiffrées doit être réalisée. C'est cet ensemble qui constitue un scenario de partage de la valeur économique.

Ces scénarisations des flux permettent ainsi de tester de manière quantitative de nombreuses hypothèses telles que la mise en place :

- de nouvelles tarifications à l'acte ;

- de nouveaux forfaits englobant plusieurs actes ;

- de forfaits rémunérant les missions de coordination (management de la structure, temps de concertation interprofessionnelle) ;

- de forfaits rémunérant l'activité d'éducation thérapeutique.

- Elles permettent également de simuler la mise en place de nouvelles conventions entre acteurs en prenant en compte :

- le développement d'activités supplémentaires pour les experts au travers de téléconsultations ou téléexpertises ;

- la substitution par des téléconsultations de déplacements d'experts vers les patients (gain de temps de transport pour les experts afin de réaliser par exemple plus d'actes) ;

- la substitution par des téléconsultations de consultations classiques de malades amenés en ambulance (gain des coûts des ambulances et du temps de personnel d'accompagnement) ;

- le gain de distance avec des transports (déplacement vers un établissement plus proche équipé de station de télémédecine) ;

- la facturation de l'éducation thérapeutique et des formations, accroissement de revenu rendu possible pour certains établissements par l'accroissement de la rapidité, du confort et de la sécurité pour le patient ;

- les changements organisationnels entrainés (diminution des hospitalisations inappropriées par les urgences, diminution des consultations programmées avec transport et diminution de la durée des hospitalisations).

\section{Etape 7 : Analyse du solde des marges pour chacune des parties prenantes}

L'étape 7 consiste à calculer le solde des marges de chacune des parties prenantes en fonction du temps. La figure 4 présente un exemple de visualisation du solde des marges de chaque partie prenante pour un scenario donné. 


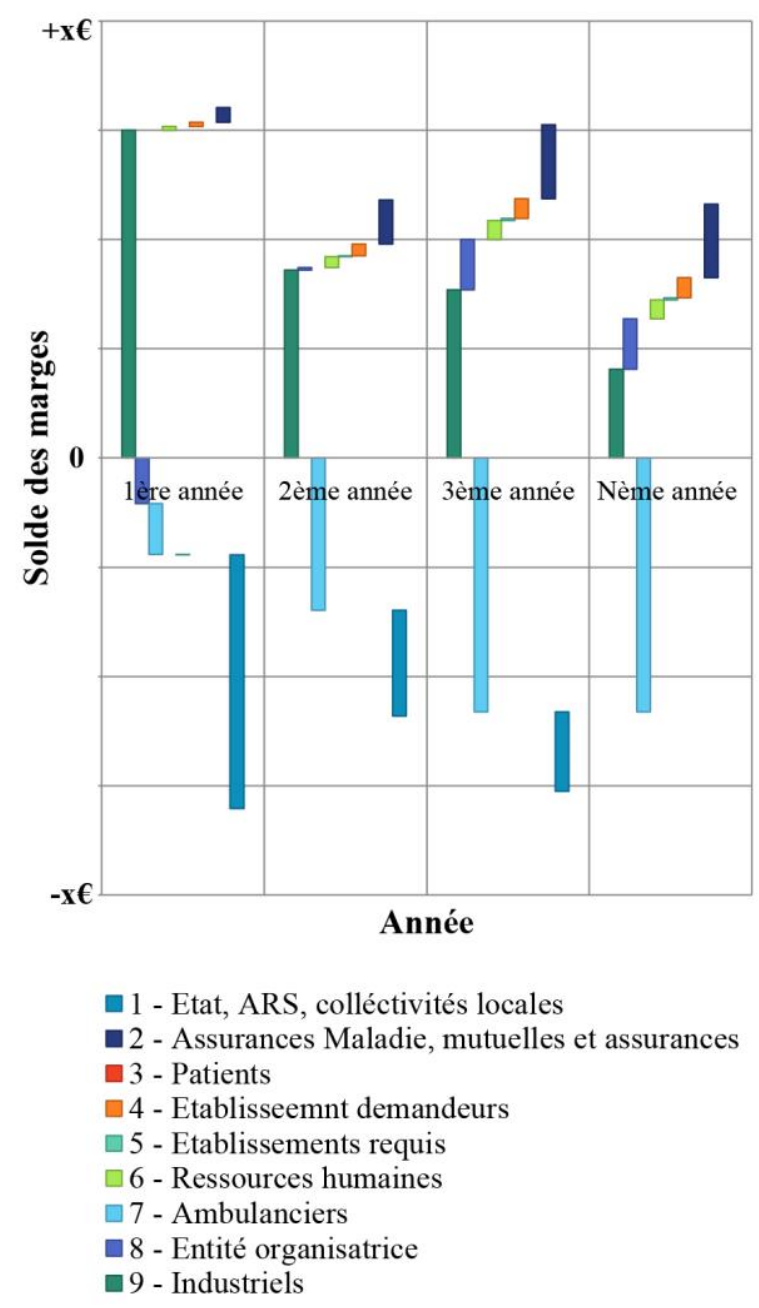

Figure 4 : Projection du solde des marges pour chaque partie prenante pour un scenario Figure 4: Projection of the balance of margins for each stakeholder for a scenario

Sur ce scenario, nous pouvons remarquer que, sur le long terme, l'ensemble des parties prenantes (mis à part les ambulanciers) ont un solde des marges positif. Cela veut dire que la valeur économique originellement allouée aux ambulanciers a été redistribuée aux autres acteurs durablement. Il y a eu un nouveau partage de la valeur. Nous pouvons ainsi faire une recommandation pour faire évoluer ce métier, par exemple vers toutes autres activités liées à la santé au domicile.

\section{Discussion}

Un des points intéressant de la méthode est de permettre à chaque partenaire de visualiser et de projeter son retour sur investissement en regard de son investissement initial et des différents modes de financements du système. Une des limites est qu'elle requiert un fort degré de concertation entre des acteurs ayant des objectifs différents.

Une autre valeur ajoutée de la méthode est de pouvoir modéliser la mise en place, la montée en charge du déploiement et la mutualisation d'une nouvelle technologie. La méthode permet également de pouvoir calculer le forfait minimal, le nombre minimal d'utilisateur ou de consultation nécessaire pour assurer la viabilité du système.

Cette méthode peut être également vue comme un outil complémentaire à la « matrice des impacts des effets attendus de la télémédecine " préconisée par la HAS en 2013 [4] pour évaluer un projet de télémédecine qui élargit les points de vue usuellement adoptés à quatre grandes catégories de parties prenantes : (a) patients, aidants et famille, (b) professionnels de santé médicaux et paramédicaux, (c) établissements de santé et 
structures de santé, (d) Etat, Assurance Maladies et autres décideurs publics et financeurs. En effet, la méthode présentée dans cet article prévoit d'étendre cet élargissement à l'ensemble des parties prenantes d'un projet. Elle permet également, en vue de l'extension d'un projet existant, de concevoir des hypothèses de futurs partages de la valeur pour que chacune des parties prenantes puissent y évaluer, de leur point de vue, leurs intérêts.

Les facteurs clés d'industrialisation de la télémédecine sont fonction de la viabilité et pérennité du modèle économique. Il est important de souligner que le succès de son déploiement dépend également de l'accompagnement à la conduite du changement car les nouvelles pratiques ont un impact important sur les relations entre professionnels [21].

\section{Conclusion}

Cet article présente une méthode en sept étapes permettant de concevoir de nouveaux partages de la valeur économique entre les parties prenantes d'un projet en fonction du temps. La méthode présentée permet de simuler et de justifier différents modèles de partage de la valeur économique grâce à une cartographie des flux existants et potentiels entre les parties prenantes du système et un calcul du solde de leurs marges respectives. Elle requiert un dialogue important entre les parties prenantes pour fixer les scenarii de partage de la valeur. Elle n'inclut qu'un aspect économique et ne permet pas évaluer la perte ou le gain de la qualité de soins liés à ce nouveau système de télémédecine. Elle est donc complémentaire des études médicoéconomiques classiques et des études multicritères. Afin de vérifier l'efficacité de cette méthode, son application nécessite d'être évaluée sur d'autres projets de télémédecine.

\section{Conflit d'intérêts}

Les auteurs déclarent ne pas avoir de conflit d'intérêts en relation avec cet article

\section{Références}

[1] Simon P, Acker D. La place de la télémédecine dans I'organisation des soins. 2008.

[2] CNOM. Télémédecine - Les préconisations du Conseil National de l'Ordre des Médecins. 2009.

[3] Lasbordes P. La télésanté: un nouvel atout au service de notre bien-être - Un plan quinquennal écoresponsable pour le déploiement de la télésanté en France. 2009.

[4] HAS. Efficience de la télémédecine état des lieux de la littérature internationale et cadre d'évaluation. 2013.

[5] Drummond MF, Sculpher MJ, Claxton K, Stoddart GL, Torrance GW. Methods for the economic evaluation of health care programmes. Oxford university press. 2015

[6] Woronoff-Lemsi M-C, Limat S, Husson M-C. Approche pharmaco-économique : évaluation pharmacomédico-économique de stratégies thérapeutiques : éléments de méthodologie. 2000.

[7] HAS. Choix méthodologiques pour l'évaluation économique à la HAS. 2011.

[8] Pascal C. La télémédecine face aux enjeux de l'évaluation médicoéconomique. European Research in Telemedicine / La Recherche Européenne en Télémédecine. 2012;1(3-4):125-9. DOI: 10.1016/j.eurtel.2012.09.002.

[9] Le Goff-Pronost M, Nassiri N. Deux approches nouvelles dans l'evaluation de la télémédecine : l'evaluation contingente et l'analyse multicritere. Cahier de recherche Marsouin. 2005(7).

[10] Kidholm K, Ekeland AG, Jensen $L K$, Rasmussen J, Pedersen CD, Bowes A, et al. A model for assessment of telemedicine applications: mast. International journal of technology assessment in health care. 2012;28(1):44-51. DOI: 10.1017/S0266462311000638.

[11] Bergmo T. Can economic evaluation in telemedicine be trusted? A systematic review of the literature. Cost Effectiveness and Resource Allocation. 2009;7(1):18. DOI: 10.1186/1478-7547-7-18. 
[12] Ekeland AG, Bowes A, Flottorp S. Effectiveness of telemedicine: A systematic review of reviews. International Journal of Medical Informatics. 2010;79(11):736-71. DOI: 10.1016/j.ijmedinf.2010.08.006.

[13] Simon HA. Les sciences de l'artificiel. Éd. rev. et complétée (2004) ed: Gallimard; 1969.

[14] Le Moigne J-L. La théorie du système général. ed: Presses Universitaires de France; 1985.

[15] Jean C. Comment réussir l'intégration de systèmes technologiques innovants au sein de systèmes complexes organisationnels? Application à la télémédecine en France [thèse]. Laboratoire Génie Industriel, Ecole centrale Paris; 2013.

[16] Porter ME. Competitive Advantage: Creating and Sustaining Superior Performance: Free Press; 1986.

[17] FranceAgriMer. Prix et coûts dans l'agroalimentaire. 2012.

[18] PIPAME. Prospective du m-tourisme. 2011.

[19] Benhamou F, Guillon O. Modèles économiques d'un marché naissant : le livre numérique. Culture prospective. 2010;2(2):1-16.

[20] Espinoza P, Gouaze A, Talange C, Bonnet B, Fabbro M, Saint-Jean O, et al. Déploiement de la télémédecine en territoire de santé. Télégéria, un modèle expérimental précurseur. Techniques hospitalières. 2011;725:9-17.

[21] Mathieu-Fritz A, Esterle L. Les transformations des pratiques professionnelles lors des téléconsultations médicales : Coopération interprofessionnelle et délégation des tâches. Revue française de sociologie. 2013;54(2):303-29. DOI : 10.3917/rfs.542.0303 